\title{
Olson vs. Coase: coalitional worth in conflict
}

\author{
Joan Esteban \\ Institut d'Anàlisi Econòmica, CSIC \\ and \\ József Sákovics \\ University of Edinburgh
}

September, 2002

\begin{abstract}
$\underline{\text { Abstract }}$
We analyze a model of conflict with endogenous choice of effort, where subsets of the contenders may force the resolution to be sequential: First the alliance fights it out with the rest and - in case they win - later they fight it out among themselves. For three-player games, we find that it will not be in the interest of any two of them to form an alliance. We obtain this result under two different scenarios: equidistant preferences with varying relative strengths, and vicinity of preferences with equal distribution of power. We conclude that the commonly made assumption of superadditive coalitional worth is suspect.
\end{abstract}

Key words: coalition formation, conflict, alliance.

JEL Numbers: D72, D74. 


\section{Introduction}

The issue of coalition formation has long been of interest to economists. A neat and useful model was developed for this purpose: games in coalitional form. The description of these games consists of a mapping from subsets of individuals to payoffs. Under the Coasian hypothesis that within any group the players would efficiently get to agreement, it is natural that it has been assumed that the payoff to the sum of two groups is no less than what the groups would obtain separately. This assumption has been central in the literature on coalition formation. In contrast with this basic assumption, there is the view held by Olson (1965) according to which the outcome of pooling efforts is sub-additive: the larger the group the more inefficient it becomes in the pursuit of its ends. This is so because the belonging to a group or a coalition does not stop individuals to act in their own private interests and hence free-ride their fellow coalition members. ${ }^{1}$ The present paper offers a first attempt at the resolution of the issue of the super- or sub-additivity of coalitional worth when individuals cannot write binding contracts.

Hence, we look at alliance formation between parties involved in conflict. The process of coalition formation is usually seen as a one-shot, rather than a sequential process. Specifically, coalitions that might themselves break up are disregarded on the basis that they are not credible. This is a persuasive argument as long as the problem is one-shot. Yet, there are many instances in which even if the partners are fully aware that heir coalition will eventually break up it is in their interest to coordinate against a third party. Examples abound. The Soviet Union and the US were allies against Nazi Germany in spite of being fully aware of their flatly opposing interests. The fall of Nazism brought the end of the alliance and the beginning of a subsequent stage of the game: the cold war. The victory of one coalition over another changes the problem altogether and may end the alliance. Yet, for both allies it might be better to keep the alliance up until they reach the stage in which the common enemy has disappeared. Thus, in this paper we explore the viability of temporary alliances that take the form of a tacit agreement between a subset of agents to postpone the resolution of their own conflict of interest and concert effort against their common opponent. Perhaps the best example for the "sequencing" of conflict that we have in mind is that of a revolution. Think

\footnotetext{
${ }^{1}$ In Esteban and Ray (2001) it is proven that when groups participate in a conflict game against each other, the win probability -contrary to Olson's claim — does increase with group size. Yet, and this is what we are interested in here, individual expected utility is inversely related to group size because of the free-riding.
} 
of practically any of them: the French, the Mexican, the Russian etc. The winning coalition almost always has broken up and victory has degenerated into further conflict. ${ }^{2}$

The second reason why we think that the standard approach is not appropriate is that it assumes that coalition members coordinate and jointly decide the action to be taken by each individual member. In this way (most of) the literature simply assumes away one of the main reasons presented by Olson (1965) as to why large groups might be ineffective in achieving their goals: the free-rider problem. A natural way of allowing to test whether the free-riding effects are sufficient to overcome any potential returns to size is by assuming that alliances merely coordinate on the common target, but that cannot write a binding agreement prescribing the actions to be taken by each member. In this paper we shall explicitly assume that each player individually chooses the amount of effort to expend. This assumption captures the behavior of political alliances, in which the allied parties do not commit to an equal level of effort. Party coalitions have the purpose of increasing the winning probabilities against the threat of a third party, without giving up on their own ideological identity. This, clearly, was the logic behind the pre World War II "popular fronts" in Europe.

The two points just made seem to correspond well to the motivating examples. Yet, they are conceptually distinct. In this paper we shall examine the role of each of the two differential aspects of behavior: temporary alliances and independent decision making.

In short, this paper should be seen as complementary to the approach taken by Ray and Vohra $(1997,1999)$ to model non-cooperative coalition formation: ${ }^{3}$ we are not looking for an agreement, in fact, we are not allowing any binding agreement; and instead of (just) looking for efficiency, we check for the viability of strategic manipulation of the game of conflict.

At the cost of restricting the analysis to three players, we can incorporate into the analysis two characteristics that are likely to influence whether coalitions form. In our first model, we analyze a contest (that is, a conflict where the players only value their favorite outcome) for any possible distribution of the size/strength of the individuals. We find that sequencing will never occur. Next, - for equal distribution of power - we show that no matter how much players value each other's favorite options, unless they completely agree sequencing does not occur in equilibrium.

\footnotetext{
${ }^{2}$ There are many economic examples as well. Two firms may decide to join forces in their research efforts to be in a better position in a patent race against a third, larger(?) competitor. In the event of their winning the race, however, they compete against each other in the product market. See, for example, Yi and Shin (2000).

${ }^{3}$ We refer the reader to their papers -- as well as to Greenberg (1994) and Bloch (1997) -- and references therein for a broader literature review. We discuss the recent literature below.
} 
Recently, a number of papers have looked at coalition formation in conflict. Skaperdas (1998) and Tan and Wang (1999) showed that, with exogenously given effort when the value/synergy function of the players is super-additive, sequencing of conflict does (for 3 players always) arise in equilibrium. As we show below, their result is not robust to endogenizing the choice of effort. That is, if the amount of effort put into conflict is a strategic variable, the moral hazard problem arising between allies can wipe out the advantages of economies of scale, turning the value function sub-additive.

Niou and Tan (1997) analyze a model similar to ours in an explicit military context. The two main differences are that their countries can sign non-agression pacts and that the winning coalition can share the loser's resources. Their results are almost diametrically opposed to ours.

Baik and Lee (2001), Bloch et al. (2002) and Noh (2002) do endogenize the outlay decisions, however they assume that there is no further conflict within the alliance. Under these assumptions coalitions may form. This is consistent with our result in Esteban and Sákovics (2000), where we show that if we allow the members of the coalition to efficiently share the surplus - in the proportion of their would-be shares in conflict - then alliances may form, at least when the third party is much weaker than the two of them together. These results indicate that the effect of free-riding can be countervailed by a decrease in aggregate cost of conflict.

The closest model to ours is Garfinkel (2002). She does allow for a strategic game of conflict both within and between the alliances (though, within an alliance she assumes that there is joint production as well as a fight for appropriation occurs), and she shows that alliances only occur in large economies. She, however, does not examine the effects of differential strength and vicinity of interest.

The paper is structured as follows. In Section 2, we present a probabilistic model of conflict with endogenous choice of effort, based on Esteban and Ray (1999). In Section 3, we treat the case of equidistant preferences and variable strengths, while in Section 4 we focus on the relevance of the vicinity of interests, with equal strength distribution. The numerical calculations were done by Mathematica and are available from the authors, or at the following URL: 


\section{A model of non-cooperative conflict}

In this section, we lay down the foundations of our model of conflictual resolution of opposing interests with endogenous choice of effort. ${ }^{4}$ In order to gain in analytical depth we shall keep the conflict model at its simplest version possible.

We shall assume that there are three individuals (or groups), $i=1,2,3$. Each individual prefers one alternative above the rest. We shall index alternatives by the name of the individual for which it is the most preferred choice. We shall thus have alternatives $j=$ $1,2,3$. We shall denote by $u_{i j}$ the valuation by individual $i$ of alternative $j$, with $u_{i i}>u_{i j}$ for all $i \neq j$. We shall assume the following valuations: $u_{i i}=1, i=1,2,3, u_{12}=u_{21}=1-v, 0$ $\leq v<1$, and $u_{i j}=0$ for all the other valuations. This simple set-up will allow us to look at the case of contest $(v=1)$ as well as the case where two players' interests are less distant than the third one's $(v<1)$.

Individuals see the outcome of the non-cooperative conflict game as probabilistic, with probabilities depending on the amount of effort contributed by the different individuals. We shall denote by $r_{i}$ the effort contributed to conflict by individual/group $i$.

To fix ideas, let us start by considering the case in which no alliance or any form of coordination among players takes place. The probability of success of the alternative pushed by player $i, p_{i}$, is assumed to be determined by

$$
p_{i}=\frac{n_{i} r_{i}}{\sum_{j=1}^{3} n_{j} r_{j}}
$$

where $n_{i}$ stands for the relative efficiency with which individual/group $i$ turns effort into effective influence on the probability distribution, with $\Sigma n_{i}=1$. Thus, $n_{i}$ can be interpreted either as the size of group $i$ or as the power of the $i$-th individual. We shall take the interpretation of the vector $\boldsymbol{n}$ as the distribution of power.

Depending on the effort he expects the other players to contribute, individual $i$ will choose the amount of effort $r_{i}$ maximizing her expected utility while deducting the utility loss produced by the effort expended, which we assume to be quadratic. That is,

$$
E u_{i=} \sum_{j=1}^{3} p_{j} u_{i j}-\frac{1}{2} r_{i}^{2}=u_{i i}-\sum_{j=1}^{3} p_{j} v_{i j}-\frac{1}{2} r_{i}^{2}
$$

\footnotetext{
${ }^{4}$ Our model is based on Esteban and Ray (1999).
} 
where $v_{i j} \equiv u_{i i}-u_{i j}>0, \forall j \neq i$. Thus, accordingly with our assumptions on preferences, we shall have $v_{i i}=0, i=1,2,3, v_{12}=v_{21}=v, 0 \leq v<1$, and $v_{i j}=1$ for all the other valuations.

Lemma 1 The pure game of conflict has a unique Nash equilibrium characterized by

$$
p_{i} \sum_{j=1}^{3} p_{j} v_{i j}-r_{i}^{2}=0, \quad i=1,2,3 .
$$

Proof: To simplify notation we shall denote by $R$ the aggregate amount of effective effort expended,

$$
R=\sum_{j=1}^{3} n_{j} r_{j}
$$

Using this notation the winning probability of alternative $i$ can be rewritten as

$$
p_{i}=\frac{n_{i} r_{i}}{\sum_{j=1}^{3} n_{j} r_{j}}=\frac{n_{i} r_{i}}{R}
$$

The first order condition for a maximum is

$$
\frac{n_{i}}{R} \sum_{j=1}^{3} p_{j} v_{i j}-r_{i}=\frac{1}{r_{i}}\left\{p_{i} \sum_{j=1}^{3} p_{j} v_{i j}-r_{i}^{2}\right\}=0, \quad i=1,2,3 .
$$

It is straightforward to check that the second order conditions are satisfied so that (5) completely characterizes the best response of an agent to the efforts contributed by the others. Therefore, the vector $r \in \mathfrak{R}_{+}^{3}$ is a Nash equilibrium of the conflict game if and only if $r_{i}$ satisfies (5) for $i=1,2,3$.

Next, define the equilibrium in terms of the vector of winning probabilities, $\boldsymbol{p}$, and the total effort expended, $R$. Let us define the $3 \times 3$ matrix $\boldsymbol{W}$ with characteristic element $w_{i j}=n_{i}^{2} v_{i j}$. Observe that we can rewrite the equilibrium conditions in matrix form as

$$
p \boldsymbol{W}=\boldsymbol{p} R^{2}
$$

Note that $\boldsymbol{p}$ is the eigenvector and $R^{2}$ the eigenvalue of the matrix $\boldsymbol{W}$. From the PerronFrobenius theorem we know that a vector $\boldsymbol{p}$ in the unit simplex and a positive number $R^{2}$ solving (7) do exist and that they are unique. Consequently, we have established that our conflict game has a unique Nash equilibrium, characterized by (5). QED

Note that using (5) we can rewrite the equilibrium expected utility of player $i$ as 


$$
\begin{aligned}
& E u_{i}=u_{i i}-\left\{\sum_{j=1}^{3} p_{j} v_{i j}+\frac{1}{2} r_{i}^{2}\right\}=u_{i i}-\left\{\sum_{j=1}^{3} p_{j} v_{i j}+\frac{1}{2} p_{i} \sum_{j=1}^{3} p_{j} v_{i j}\right\}= \\
& =u_{i i}-\frac{2+p_{i}}{2} \sum_{j=1}^{3} p_{j} v_{i j}
\end{aligned}
$$

\section{Strategic alliances in contests}

In this section we assume that the players only attach positive value to their preferred alternative, while they are indifferent between the others. This restriction to "contests" enables us to analyze the problem for any arbitrary distribution of power. We proceed by first characterizing the equilibrium utilities that the players expect in a contest. Next, we turn to the calculation of the equilibrium expected utilities of a game in which an alliance is formed. We do this via backward induction, first calculating the equilibrium of the conflict game that arises in case the alliance comes out victorious from the first stage. Then, using these continuation values, we resolve the conflict game of the first stage.

In the case of contests, the expected utility of agent $i$ is given by

$$
u_{i}=p_{i}-\frac{1}{2} r_{i}^{2}
$$

The first order condition (5) can now be written as

$$
\left(1-p_{i}\right) \frac{n_{i}}{R}=r_{i} \text {. }
$$

Multiplying both sides of (9) by $\frac{n_{i}}{R}$ and rearranging we have

$$
p_{i}=\frac{\left(\frac{n_{i}}{R}\right)^{2}}{1+\left(\frac{n_{i}}{R}\right)^{2}} .
$$

By (9) and (10),

$$
p_{i}\left(1-p_{i}\right)=r_{i}^{2}
$$

and thus the equilibrium ex-ante expected utility, (8), becomes,

$$
u_{i}=p_{i}-\frac{1}{2} p_{i}\left(1-p_{i}\right)=\frac{1}{2} p_{i}\left(1+p_{i}\right) .
$$

In equilibrium, condition (10) has to hold for every $i=1,2,3$. Hence, adding over all players, and taking into account that $\Sigma p_{i}=1$, we have the (unique) equilibrium value of $R$ implicitly determined by the equation, 


$$
\sum_{i} \frac{\left(\frac{n_{i}}{R}\right)^{2}}{1+\left(\frac{n_{i}}{R}\right)^{2}}=1
$$

Plugging this value of $R$ into (10) we can obtain the equilibrium vector of probabilities, $\boldsymbol{p}$.

Without loss of generality, we will adopt the following parametrization: $n_{1}=1-n, n_{2}$ $=n m$ and $n_{3}=n(1-m)$. We are going to consider the possible formation of an alliance between players 2 and 3 . These two players can alter the game by forcing the conflict game to be resolved via two sub-conflicts. If the alliance is formed, then in the first round the partners will jointly fight against player 1 . That is, the first sub-conflict will be described by a lottery, which either gives 1 or the alliance of 2 and 3 as the winner. The alliance members choose their outlay separately, as a function of their expectations in the second sub-conflict. This subgame only arises if the alliance wins in the first conflict, in which case the partners have to fight it out between themselves.

Let us start our analysis with the resolution of this second stage. Using (10), (12) and (13) we can easily obtain that the expected second-stage utility of members of the allied party, $\left(w_{2}, w_{3}\right)$, will be

$$
w_{2}=\frac{m(1+m)}{2} \text { and } w_{3}=\frac{(2-m)(1-m)}{2} .
$$

Given these values, together with $w_{1}=1$, we can examine the first-stage behavior of the players. The expected utilities as a function of their outlays are

$$
\begin{aligned}
& u_{1}\left(r_{1}\right)=p_{1}-1 / 2 r_{1}^{2}, u_{2}\left(r_{2}\right)=\left(1-p_{1}\right) w_{2}-1 / 2 r_{2}^{2}, \\
& u_{3}\left(r_{3}\right)=\left(1-p_{1}\right) w_{3}-1 / 2 r_{3}^{2} .
\end{aligned}
$$

Just as in Section 2, using the first-order conditions we obtain that

$$
u_{1}=\frac{p_{1}\left(1+p_{1}\right)}{2}, u_{2}=\left(1-p_{1}\left(1+\frac{p_{2}}{2}\right)\right) w_{2}, u_{3}=\left(1-p_{1}\left(1+\frac{p_{3}}{2}\right)\right) w_{3}
$$

where $p_{2}$ and $p_{3}$ are the probabilities of winning in case the players expended efforts thinking they were sequencing the conflict but in fact it was resolved in one stage. That is, $p_{2}=\frac{n m r_{2}}{R}$ and $p_{3}=\frac{n(1-m) r_{3}}{R}$. Solving for the $p$ 's is again sufficient to determine the expected utilities in equilibrium. Let $\left(\frac{p_{i}}{r_{i}}\right)^{2}=z_{i}$. Then, again using the first-order conditions, we obtain that, 


$$
p_{1}=\frac{z_{1}}{1+z_{1}}, p_{2}=z_{2} w_{2} p_{1} \text { and } p_{3}=z_{3} w_{3} p_{1}
$$

Since again the $p$ 's add up to one, we can solve for $z_{1}$, using the fact that

$$
z_{2}=\left(\frac{\mathrm{nm}}{1-\mathrm{n}}\right)^{2} z_{1} \text { and } z_{3}=\left(\frac{\mathrm{n}(1-\mathrm{m})}{1-\mathrm{n}}\right)^{2} z_{1}
$$

We obtain

$$
z_{1}=\frac{1-n}{n \sqrt{m^{2} w_{2}+(1-m)^{2} w_{3}}}
$$

Let us set $\alpha=\frac{1-n}{n}$ and $A=m^{3}(1+m)+(1-m)^{3}(2-m)$. Substituting (17) and (18) back into (16) and using the previously computed continuation values, we then have

$$
p_{1}=\frac{\alpha \sqrt{2 A}}{A+\alpha \sqrt{2 A}}, \quad p_{2}=\frac{m^{3}(1+m)}{A+\alpha \sqrt{2 A}} \quad \text { and } \quad p_{3}=\frac{(1-m)^{3}(2-m)}{A+\alpha \sqrt{2 A}} .
$$

Finally, substituting back into (15) we obtain the expected utility of each player:

$$
\begin{gathered}
u_{1}=\frac{\alpha \sqrt{2 A}(A+2 \alpha \sqrt{2 A})}{2(A+\alpha \sqrt{2 A})^{2}} \\
u_{2}=\frac{\alpha \sqrt{2 A}\left(2 A-m^{3}(1+m)\right)+2 A^{2}}{2(A+\alpha \sqrt{2 A})^{2}} \cdot \frac{m(1+m)}{2} \\
u_{3}=\frac{\alpha \sqrt{2 A}\left(2 A-(1-m)^{3}(2-m)\right)+2 A^{2}}{2(A+\alpha \sqrt{2 A})^{2}} \cdot \frac{(1-m)(2-m)}{2} .
\end{gathered}
$$

Following these same steps for the benchmark anarchy case, we can calculate the equilibrium expected utilities in total conflict. Unfortunately, the formulas are way too long to enable us to compare them directly with the utilities expected from sequenced conflict. Instead, we performed a numerical analysis and present here the plotted values of the difference between the expected utilities under the two modes of conflict.

Based on numerical analysis, we can establish the following results:

Proposition 1 In a society made up of three players/groups,

i) No two players will ever coincide in preferring to form a coalition;

ii) The sum of the utilities of the hypothetical partners is higher in anarchy;

iii) The player facing the coalition always profits from sequencing; 
iv) When either the joint power share of the hypothetical partners is small (approx. less than .2) or they are of similar power with a not too small third group, sequencing would be welfare improving.

\section{INSERT FIGURE 1 ABOUT HERE}

Figure 1 illustrates the first statement. The dark area corresponds to the parameter values for which player 2 prefers sequencing through an alliance with player 3 over playing the original one-shot, anarchic conflict game. Since the figure for player 3 is the mirror image of this one with respect to $m=.5$, it can be observed directly that there are no parameters for which both players would coincide in their wish to form an alliance. In principle, this does not rule it out that their joint utility might be higher with sequencing for some distribution of power. The second statement of the proposition shows that this is not the case, though (we omit the plot). Since the main effect of alliance formation is that the outlays in the first period are reduced, we obtain that the player that is facing the alliance always profits from such a change. This profit differential is strong enough to overcome the utility decrease of the alliance for an important range of parameters. Thus, as Figure 2 shows, the joint surplus of society would increase with sequencing in about half the possible cases.

\section{INSERT FIGURE 2 ABOUT HERE}

\section{Strategic alliances with vicinity of interests}

In the previous section, we have shown that alliances do not come about based on group sizes. We shall now examine whether alliances may arise when players are of equal power, but the candidates to ally have their interests closer than to the third contending party. To facilitate the distinction with respect to the previous model, in this section we shall call the three players $i, j$ and $k$. We thus assume that $n_{h}=1 / 3, h=i, j, k$. Recall that $u_{j k}=u_{k j}=1-v$.

Let us start again with the valuation that each of the allied players will attach to the continuation of the game into its second stage. Observe first that, the two groups being symmetric, the equilibrium winning probabilities will be identical, that is, one half. This in turn implies that the per capita effort contributed by each party will be equal. Bearing this in mind, we can easily deduce from the first order condition (5) that

$$
r_{j}=r_{k}=\frac{\sqrt{v}}{2},
$$

and that the equilibrium utilities of this second stage of the game, $w_{j}$ and $w k$, are 


$$
w_{j}=w_{k}=\frac{8-5 v}{8}=w
$$

Notice that

$$
.375 \leq w \leq 1 .
$$

Let us now move on to the first stage of the game when $j$ and $k$ oppose player $i$. If the members of the alliance win, they obtain an (expected) utility $w$ given by (22) and zero otherwise. As for player $i$, the utility of winning is 1 and zero otherwise.

The expected utility by player $j$ (resp. $k$ ) is given by

$$
E u_{j}=p w-\frac{r_{j}^{2}}{2}=\frac{r_{j}+r_{k}}{r_{j}+r_{k}+r_{i}} w-\frac{r_{j}^{2}}{2},
$$

and the utility expected by player $i$ is given by

$$
E u_{i}=(1-p)-\frac{r_{i}^{2}}{2}=\frac{r_{i}}{r_{j}+r_{k}+r_{i}}-\frac{r_{i}^{2}}{2} .
$$

The best responses by players $j$ (resp. $k$ ) and $i$ can be obtained from the maximization of (24) and (25)

$$
\frac{(1-p) w}{R}=r_{j}, \text { and } \quad \frac{p}{R}=r_{i}
$$

By symmetry, $r_{j}=r_{k}$ and hence $\frac{r_{j}}{R}=\frac{r_{k}}{R}=\frac{p}{2}$. We can thus rewrite (26) as

$$
2(1-p) w=p R^{2}, \text { and } \quad p=(1-p) R^{2} .
$$

From (27) we can obtain the equilibrium winning probability of the coalition, $p$, as

$$
p=\frac{\sqrt{2 w}}{1+\sqrt{2 w}} .
$$

Using (6), we can write the equilibrium utilities of players $j$ (resp. $k$ ) and $i$ as

$$
\begin{aligned}
& E u_{j}=E u_{k}=p \frac{3+p}{4} w, \text { and } \\
& E u_{i}=\frac{(2-p)(1-p)}{2}
\end{aligned}
$$

The expected utility $E u_{j}$ is increasing in $w$ (and hence decreasing in $v$ ). Since $.375 \leq w$ $\leq 1$, we have that the equilibrium utility is lowest when $v=1$ and highest when $v=0$, that is,

$$
0.15072 \leq E u_{\mathrm{j}} \leq 0.52513
$$


Let us now obtain the equilibrium utilities of players $i, j$ and $k$ in the one-shot all-out conflict game. From (5) we can obtain the first order conditions for the best response of each player,

$$
\begin{aligned}
& \frac{p_{k} v+p_{i}}{R}=r_{j} \\
& \frac{p_{j} v+p_{i}}{R}=r_{k} . \\
& \frac{p_{j}+p_{k}}{R}=r_{i}
\end{aligned}
$$

Adding up, and bearing in mind that the probabilities add up to 1, we have

$$
(1+v)\left(1-p_{i}\right)+2 p_{i}=3 R^{2} .
$$

Notice now that condition (32) for player $i$ can be written as

$$
1-p_{i}=3 p_{i} R^{2}
$$

From (33) and (34) we can solve for $p_{i}$ and obtain,

$$
\begin{aligned}
& p_{i}=\frac{\sqrt{8+v^{2}}-(2+v)}{2(1-v)}, \text { and } \\
& p_{j}=p_{k}=\frac{1-p_{i}}{2}=\frac{4-v-\sqrt{8+v^{2}}}{4(1-v)} .
\end{aligned}
$$

The corresponding equilibrium expected utilities $E u^{*}$ will thus be

$$
\begin{aligned}
& E u_{j}^{*}=E u_{k}^{*}=1-\left(p_{k} v+p_{i}\right) \frac{2+p_{j}}{2}, \text { and } \\
& E u_{i}^{*}=\frac{p_{i}\left(1+p_{i}\right)}{2} .
\end{aligned}
$$

The equilibrium expected utility for both players $j$ and $k$ is again monotonic in $v$. It can be readily computed that

$$
E u_{j}^{*}\left(E u_{k}^{*}\right) \rightarrow 0.22222 \text { as } v \rightarrow 1 \text { and } E u_{j}^{*}\left(E u_{k}^{*}\right) \rightarrow 0.52513 \text { as } v \rightarrow 0 .
$$

We then have the following result:

Proposition 2 In a society made up of three players of equal power,

i) Independently of the closeness of interests of any two players, it will never be in the interest of either of them to form a coalition (though this difference vanishes in the limit as their interests converge);

ii) The player facing the coalition always profits from sequencing and his benefit is increasing in the distance between the preferences of the coalitional partners; 
iii) Social welfare is higher with sequencing if and only if the distance between the preferences of the coalitional partners is sufficiently high ( $v>.83$ approx.).

The three results are illustrated (and proven) in Figures 3-5:

\section{INSERT FIGURES 3-5 ABOUT HERE}

We have plotted the expected utility of player $j$ in both cases, under the sequenced game and under anarchy, in Figure 3. In view of (31) and (39) it is immediate that, no matter how close their interests are, players $j$ and $k$ do not obtain a higher equilibrium utility in the sequenced game than what they would obtain in the one-shot all-out game. In fact, sequencing makes them strictly worse off, unless their preferences coincide, in which case there is no meaningful second stage game and therefore the payoffs are independent of sequencing or not.

In Figure 4 we have the outsider's expected payoff. The lower curve corresponds to the total conflict situation. In this case, the outsider prefers the other players to have close interests, since that reduces their willingness to fight (they free ride). In the case of sequencing, the outsider's payoff is increasing in the distance between the preferences of the coalitional partners. This is due to the fact that the farther apart their preferences the less the allied players value winning in the first stage. Therefore, they further reduce their outlays, benefiting the outsider.

Finally, in Figure 5 we have depicted the social surplus. Welfare is decreasing in the distance of preferences, just as the first best does. As we can see the gains of the outsider offset the losses of the coalitional partners whenever $v$ is sufficiently large. 


\section{$\underline{\text { References }}$}

Baik, K-H., and S. Lee (2001), "Strategic groups and rent dissipation," Economic Inquiry, $39(4), 672-684$.

Bloch, F. (1997), "Non-cooperative models of coalition formation in games with spillovers," in New directions in the economic theory of the environment (Carraro and Sinischalco eds.) Cambridge University Press.

Bloch, F., Sánchez-Pagés, S. and R. Soubeyran (2002), "When does universal peace prevail? Secession and group formation in rent seeking contests and policy conflict," mimeo, GREQAM, Marseille.

Esteban, J. and D. Ray (1999), "Conflict and Distribution," Journal of Economic Theory, 87, $379-415$.

Esteban, J. and D. Ray (2001), "Free Riding and the Group Size Paradox", American Political Science Review 95, 663-672.

Esteban, J. and J. Sákovics (2000), “Temporary Alliance Formation: Endogenous Sequencing in Conflict Games," Discussion Paper, Instituto de Estudios Económicos de Galicia Pedro Barrié de la Maza.

Garfinkel, M.R. (2002), "Stable alliance formation in distributional conflict," mimeo, University of California at Irvine, June.

Greenberg, J. (1994), "Coalition structures," Chapter 37 in Handbook of Game Theory with Economic Applications (Aumann and Hart eds.), North Holland, Elsevier Science.

Niou, E. and G. Tan (1997), “A theory of alliance formation," mimeo, Duke University, August.

Noh, S.J. (2002), "Resource distribution and stable alliances with endogenous sharing rules," European Journal of Political Economy, 18, 129-151.

Olson, M. (1965), The logic of collective action, Harvard University Press.

Ray, D. and R. Vohra (1997), "Equilibrium Binding Agreements," Journal of Economic Theory, 73(1), 30-78.

Ray, D. and R. Vohra (1999), "A Theory of Endogenous Coalition Structures," Games and Economic Behavior, 26, 286-336.

Skaperdas, S. (1998), "On the formation of alliances in conflict and contests," Public Choice, $96,25-42$.

Tan, G. and R. Wang (1999), "Endogenous Coalition Formation in Rivalry," mimeo, University of British Columbia, April.

Yi, S-S. and H. Shin (2000), "Endogenous formation of research coalitions with spillovers," International Journal of Industrial Organization, 18, 229-256. 


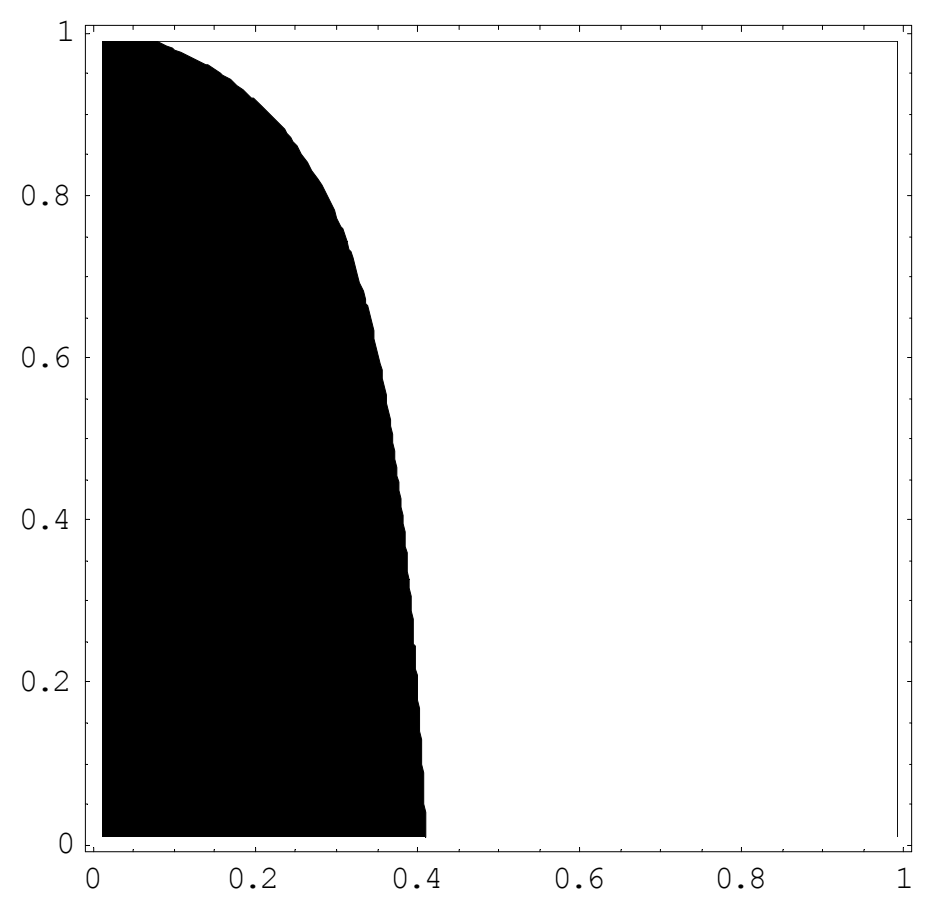

Figure 1

The size distributions for which group 2 prefers sequencing (shaded area)

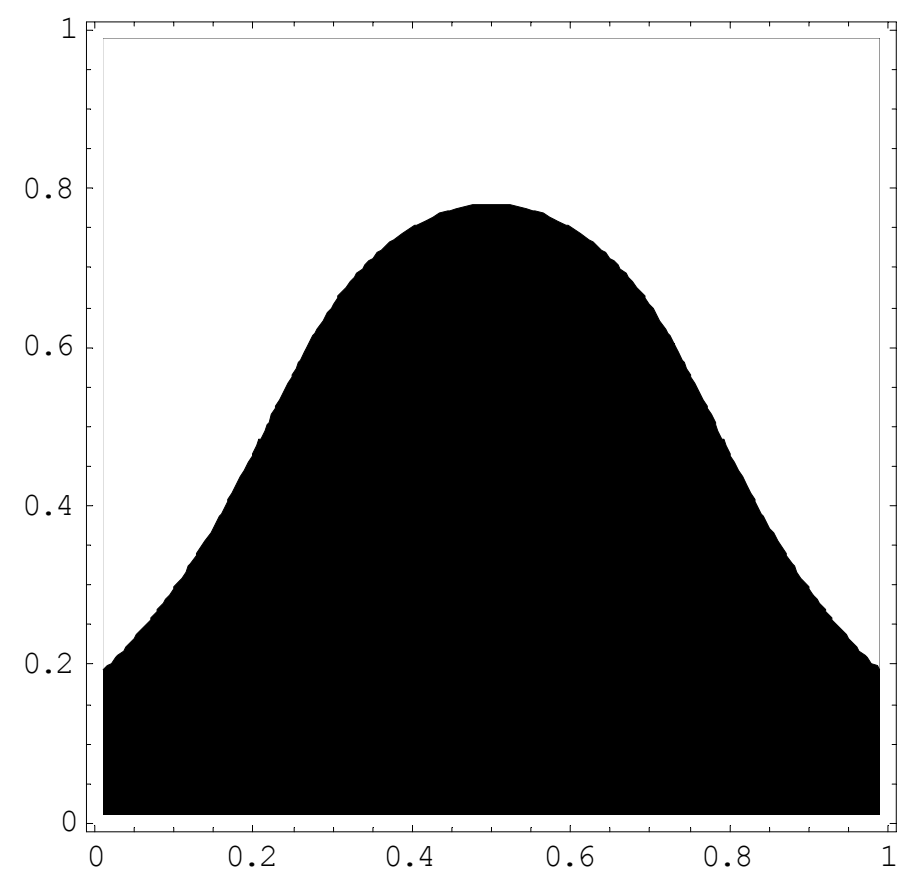

Figure 2

The size distributions for which sequencing would be socially optimal (shaded area) 


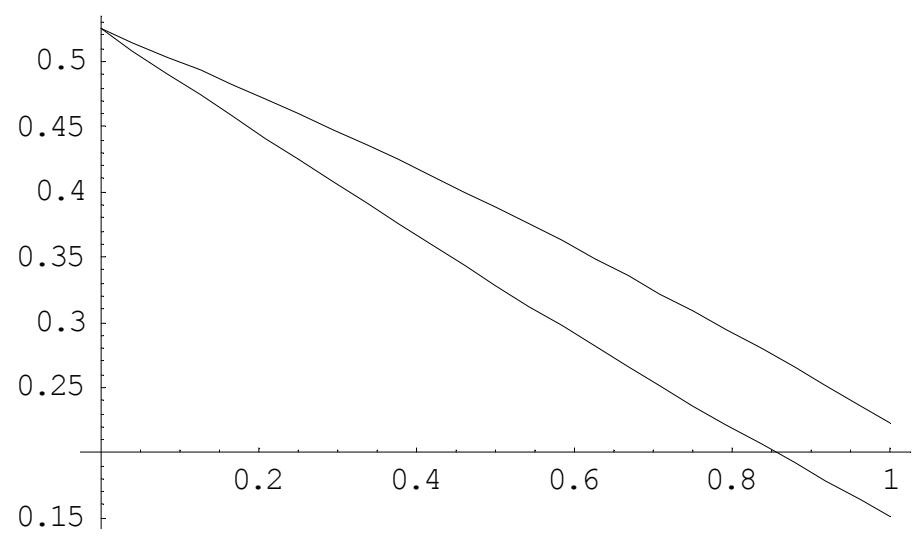

Figure 3

The expected utilities of the allied under the two regimes as a function of $v$

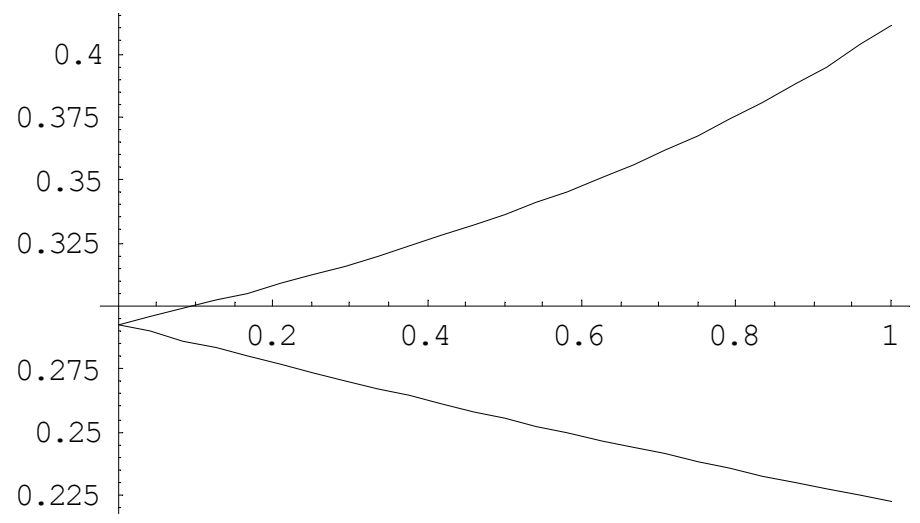

Figure 4

The expected utilities of the outsider under the two regimes as a function of $v$

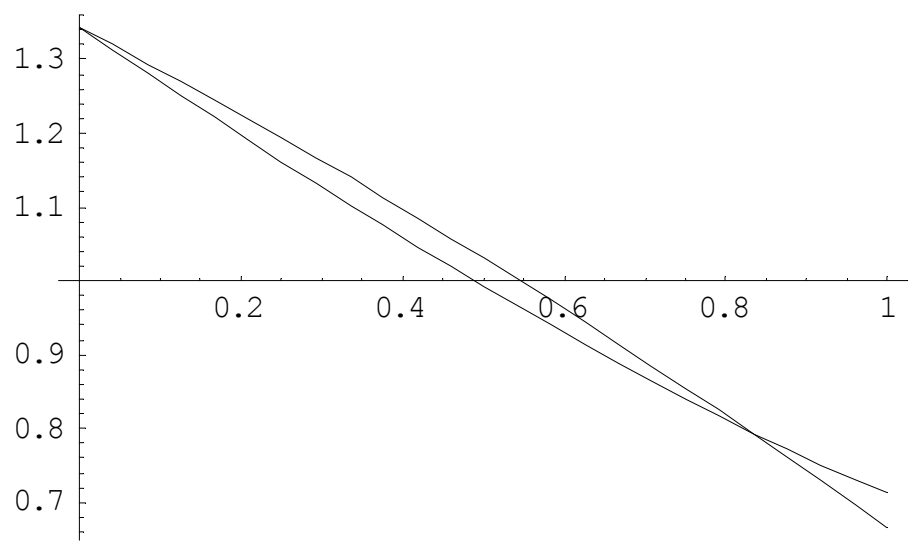

Figure 5

The social surplus under the two regimes as a function of $v$ 